\title{
Active Piezoelectric Vibration Control of Subscale Composite Fan Blades
}

\author{
Kirsten P. Duffy - University of Toledo \\ Benjamin B. Choi - NASA Glenn Research Center \\ Andrew J. Provenza - NASA Glenn Research Center \\ James B. Min - NASA Glenn Research Center \\ Nicholas Kray - GE Aviation
}




\section{Background}

- High performance fan blades

- High excitation levels

- Vibratory stresses $\rightarrow$ fatigue

- Incorporate damping into blades

- Piezoelectric materials

- Passive damping - e.g. shunt circuit

- Active vibration control

- Spin testing with active control

- Surface-mounted sensors and actuators

- Control $1^{\text {st }}$ bending vibration

- Possibility of embedding into blades

- Protect from airflow and debris

- Future testing 


\section{Piezoelectric Damping Research}

- Basic Research

- Chopra (2002) - survey of smart structures

- Hagood and von Flotow (1991) - analysis of piezoelectric damping

- Lesieutre (1998) - types of passive damping shunt circuits

- Turbomachinery Application

- Cross and Lewis (2002) - smart materials for future engines

- Cross and Fleeter (2002) - stator blade damping with passive shunt circuit

- Remington et al. (2003) - stator blade actuation for noise control

- Watanabe et al. (2008) - blade flutter control in a linear transonic cascade

- Struzik and Wang, Yu and Wang $(2007,2009)$ - piezoelectric circuits for mistuning and damping

- Hohl et al. (2009) - bladed disk model with shunt circuits - analysis and testing

- Kauffman and Lesieutre (2010) - frequency-switching for resonance avoidance

- Implementation

- Hilbert et al. (2001) - patent for shunted piezoelectric damping of blades

- Duffy et al. (2009) - piezoelectric plate damping under rotation

- Siemann et al. (2009) - piezoelectric actuation of compressor blades under rotation

- Bachmann et al. (2010) - pre-compressing piezoelectric elements to reduce centrifugal tensile stress

- Duffy et al. (2012) - effects of embedding on composite strength 


\section{Piezoelectric Vibration Control}

- $K=$ "generalized electromechanical coupling"

- Damping is proportional to $K^{2}$

- $K^{2}=$ energy converted by the piezoelectric material into electrical energy divided by the system modal strain energy

- Centrifugal effects:

- Centrifugal stiffening may increase resonance frequency, decreasing $K$

- Modal stress contours will also change with rotational speed, affecting $K$

- Tensile stress in the piezoelectric material due to spinning

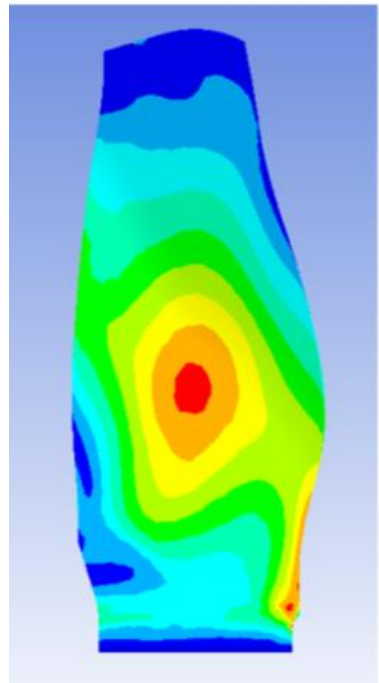

1B modal strain

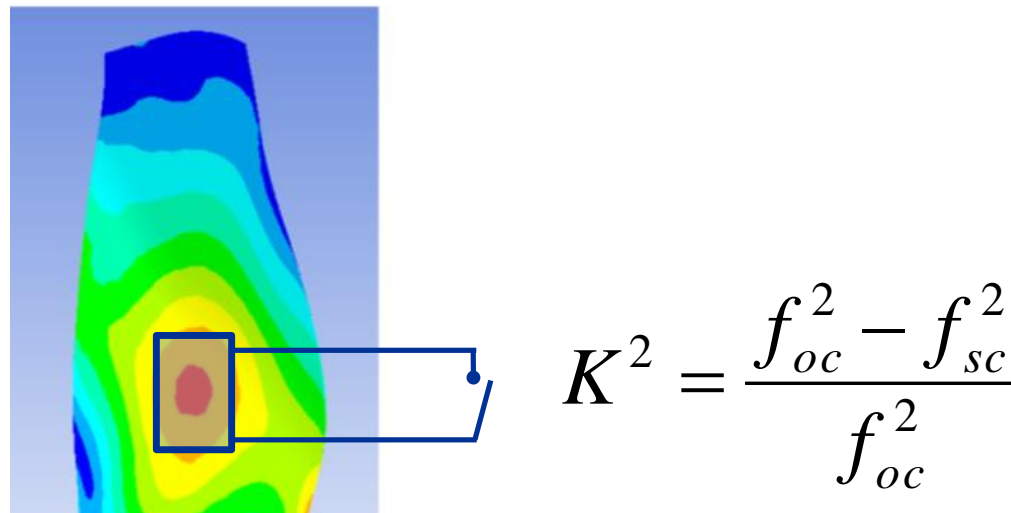

Piezoelectric element at high strain location 


\section{Test Configuration}

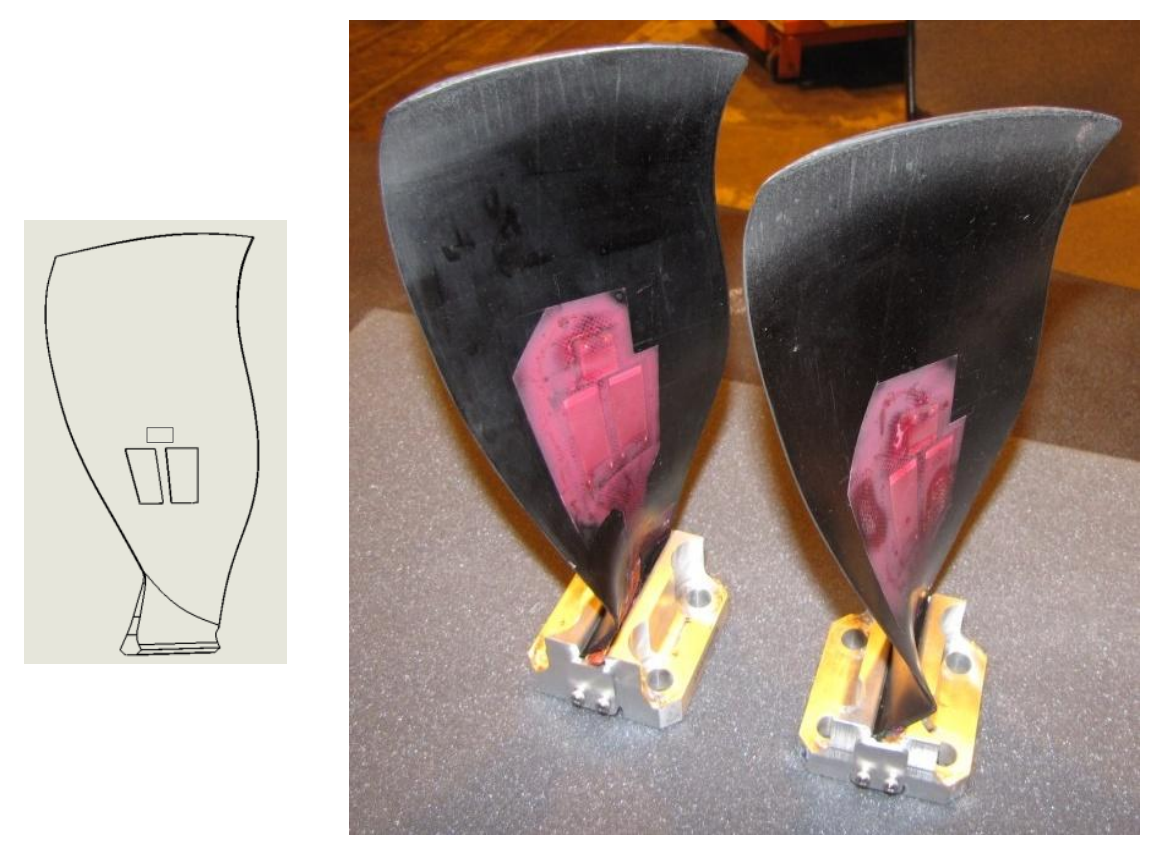

- Two actuators

- One sensor

- Located at high modal strain location for $1 \mathrm{~B}$ mode

- Expected centrifugal strain at max speed of 5000 RPM is $300 \times 10^{-6} \mathrm{~m} / \mathrm{m}$
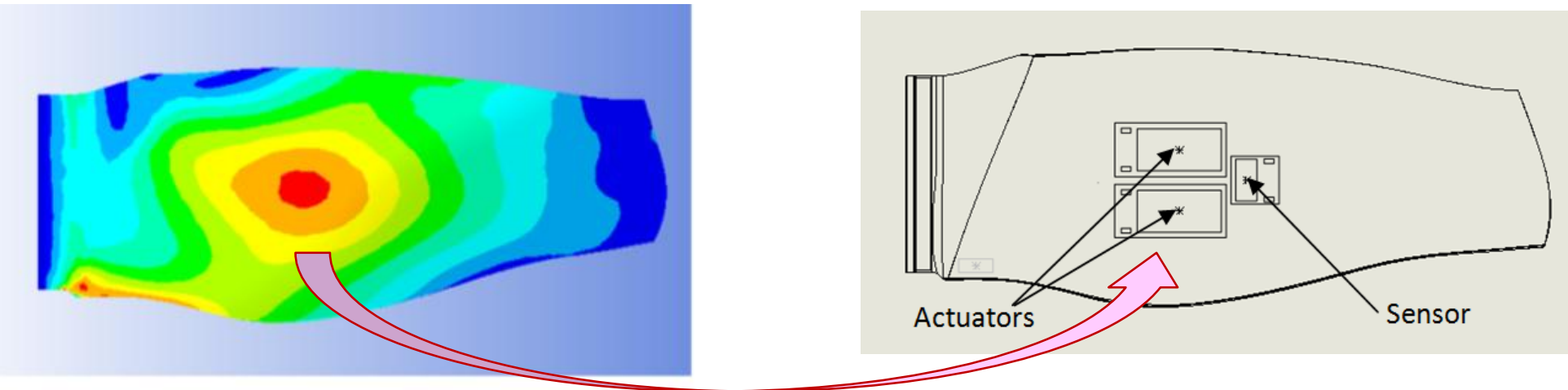


\section{Test Articles}

\begin{tabular}{|c|c|c|}
\hline Blade Material & Type & Description \\
\hline $\begin{array}{l}\text { Polymer matrix fiber } \\
\text { composite }\end{array}$ & $\begin{array}{c}\text { HexPly } 8551-7 \\
\text { with IM } 7 \text { carbon fibers }\end{array}$ & $\begin{array}{l}\text { Epoxy resin with unidirectional } \\
\text { carbon fibers, ply stack-up }\end{array}$ \\
\hline Piezoelectric Materials & Type & Description \\
\hline \multirow{2}{*}{$\begin{array}{c}\text { Flexible, } \\
\text { macro-fiber composite, } \\
\mathrm{d}_{31} \text {-type, } 300 \mathrm{~mm} \\
(0.012 ”) \text { thick } \\
\text { PZT-5A } \\
\text { (Navy Type-II PZT) }\end{array}$} & $\begin{array}{l}\text { Smart-Material Corp. } \\
\text { Sensor: } \\
\text { M-0714-P2 Qty:1 }\end{array}$ & $\begin{array}{c}14.0 \mathrm{~mm} \times 7.0 \mathrm{~mm}\left(0.55^{\prime \prime} \times 0.28 ”\right) \\
6.5 \mathrm{nF} \text { nominal capacitance } \\
-600 \times 10^{-6} \text { free strain } \\
-85 \mathrm{~N}(-19 \mathrm{lbf}) \text { blocking force }\end{array}$ \\
\hline & $\begin{array}{l}\text { Smart-Material Corp. } \\
\text { Actuators: } \\
\text { M-2814-P2 Qty: } 2\end{array}$ & $\begin{array}{c}14.0 \mathrm{~mm} \times 28.0 \mathrm{~mm}\left(0.55 " \times 1.10^{\prime \prime}\right) \\
25.7 \mathrm{nF} \text { nominal capacitance } \\
-700 \times 10^{-6} \text { free strain } \\
-85 \mathrm{~N}(-19 \mathrm{lbf}) \text { blocking force }\end{array}$ \\
\hline
\end{tabular}

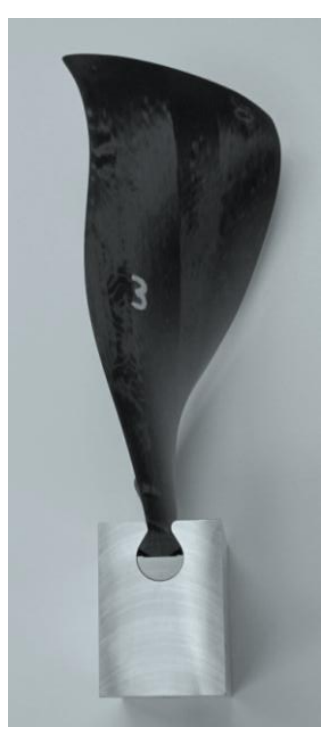

GEnx Subscale Composite Fan Blade

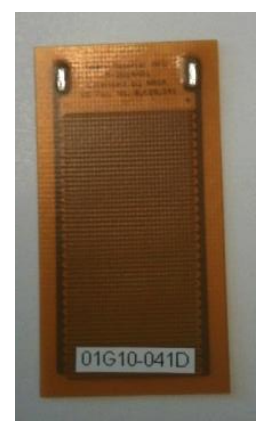

Piezoelectric Actuator 


\section{Dynamic Spin Rig Facility}

- Two blades place opposite each other in dovetail fixtures

- Vacuum

- Excitation provided by magnetic bearings to the rotor

- Slip ring

- 0-5000 RPM for this test

- Instrumentation

- Piezoelectric sensor on each blade

- Two piezoelectric actuators on each blade

- Endevco model 25A accelerometer on blade fixture

- Equipment

- Data Physics SignalCalc Mobilyzer provided excitation voltage, also measured response from sensors

- dSPACE control system

- Midé Piezoelectric amplifiers 


\section{Dynamic Spin Rig Facility}
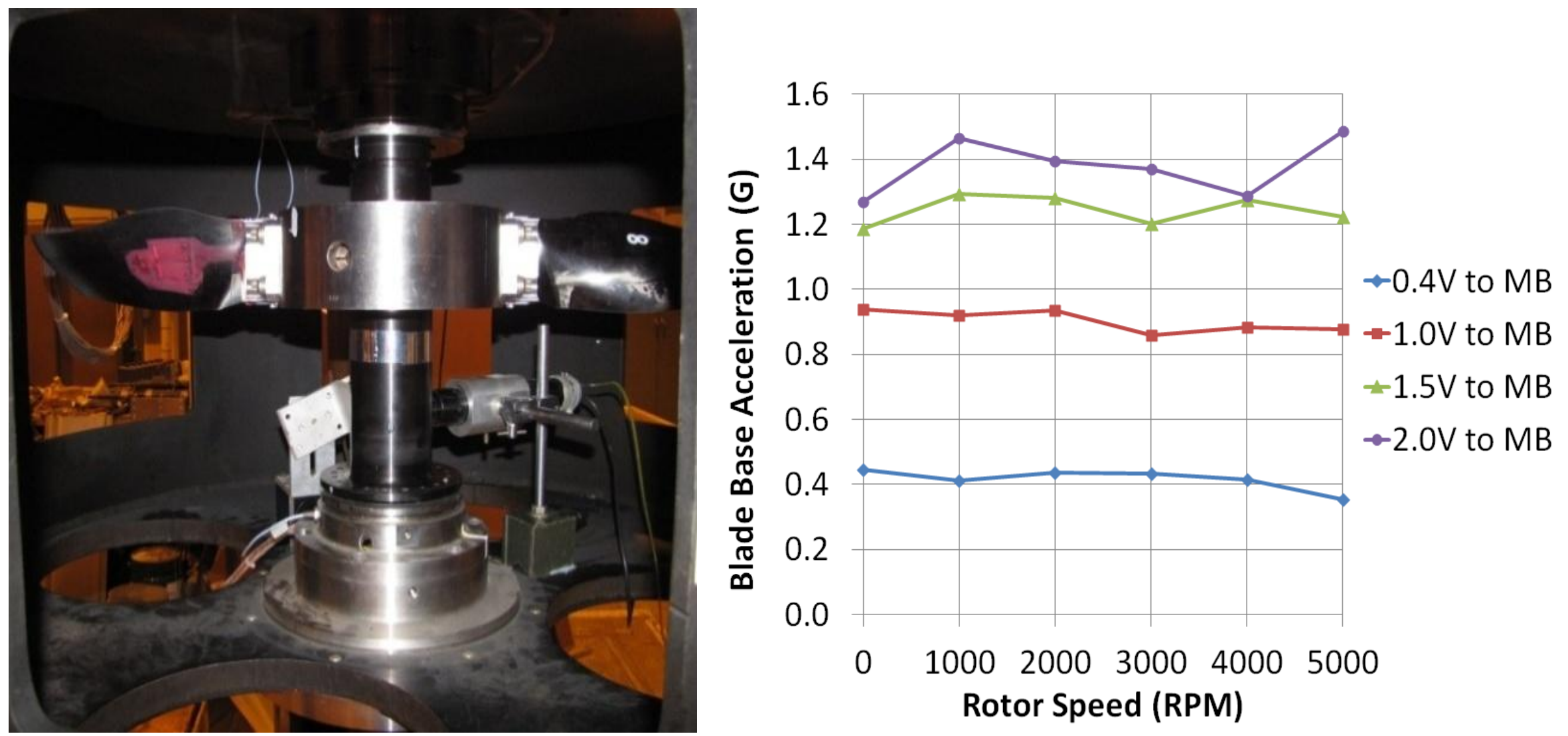


\section{Spin Test}

- Piezoelectric Sensor

- Measure response to magnetic bearing excitation - no control

- Piezoelectric Actuator

- Measure response to piezoelectric actuator excitation - no control

- Open Loop Control

- Magnetic bearing excitation

- Piezoelectric actuator at same frequency as excitation, phase chosen to reduce blade response

- Closed Loop Control

- Based on a tuned RLC circuit (Choi 2008)

- Implemented in dSPACE control code

- Amplified signal (from amplifier and within control code) 


\section{Passive RLC Shunt Circuit}

- Closed-loop control based on RLC circuit

- Capacitance C

- Piezoelectric material property

- Inductance L

- Sets the electric circuit frequency

- Resistance R

- Dissipates energy $\rightarrow$ damping

- Sets the bandwidth

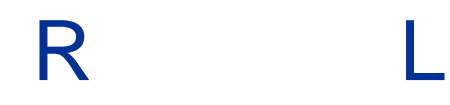

M 


\section{Closed-Loop Control System}

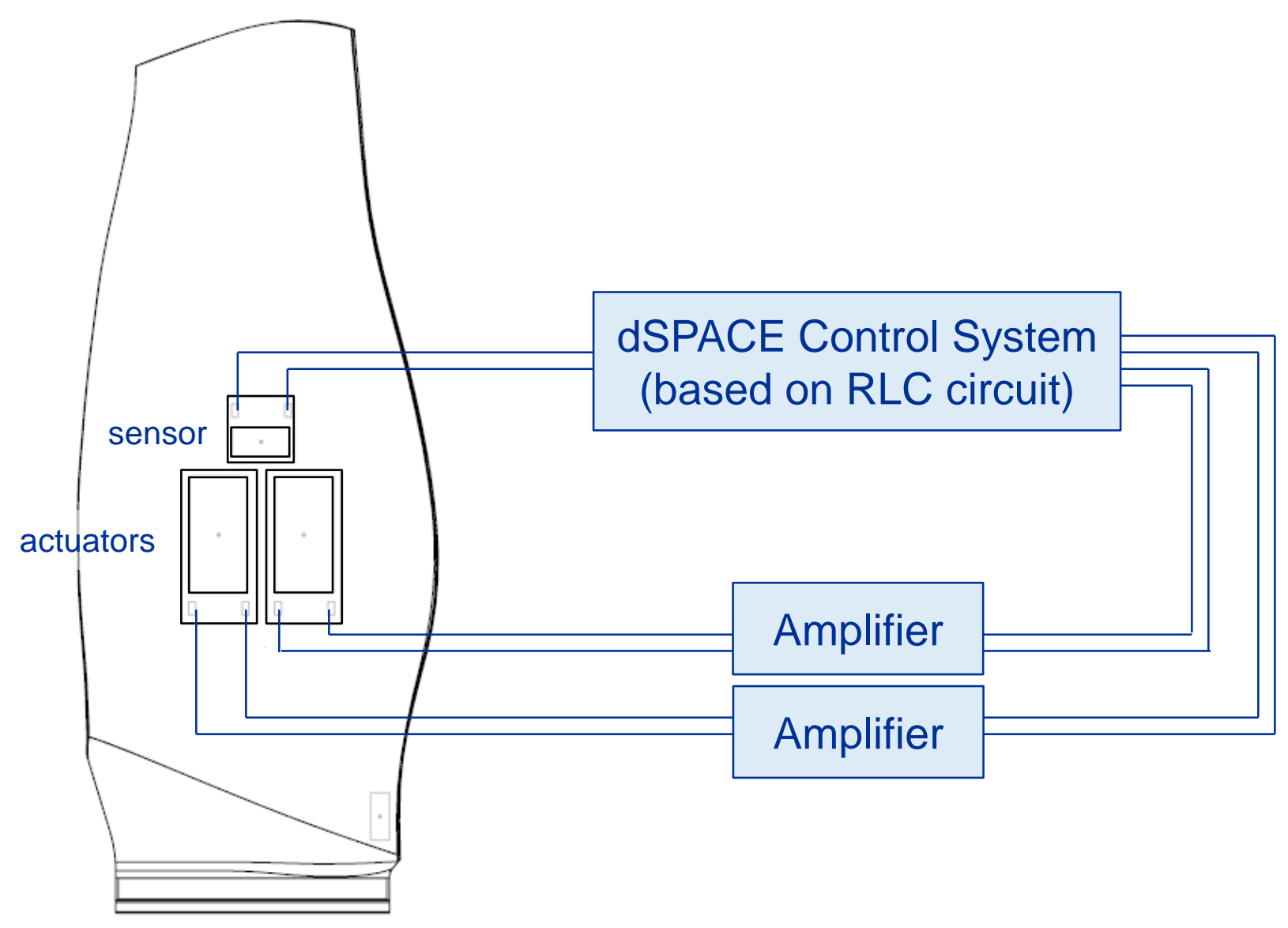




\section{Blade Resonance Frequency/Damping}

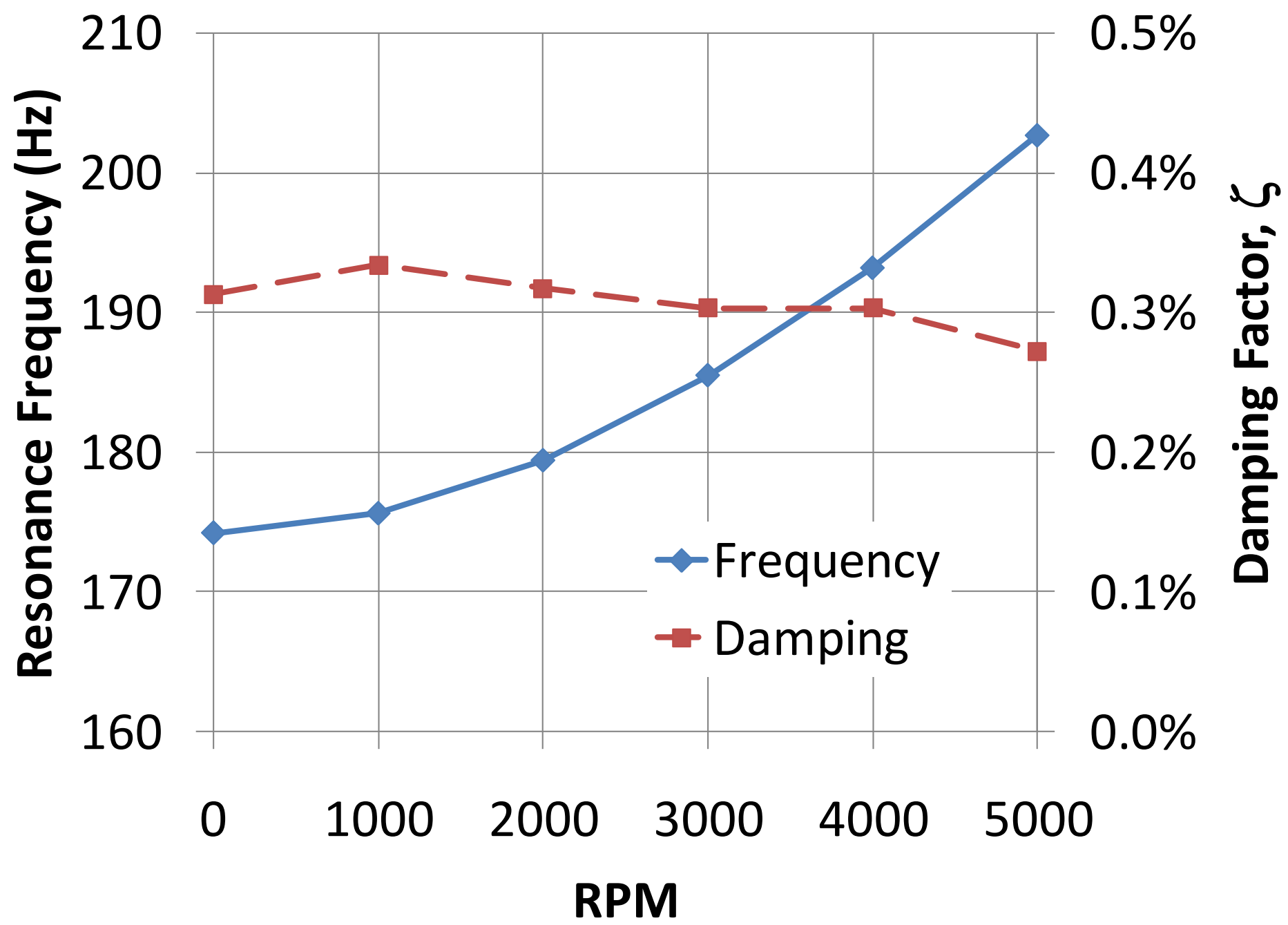




\section{Generalized Electromechanical Coupling}

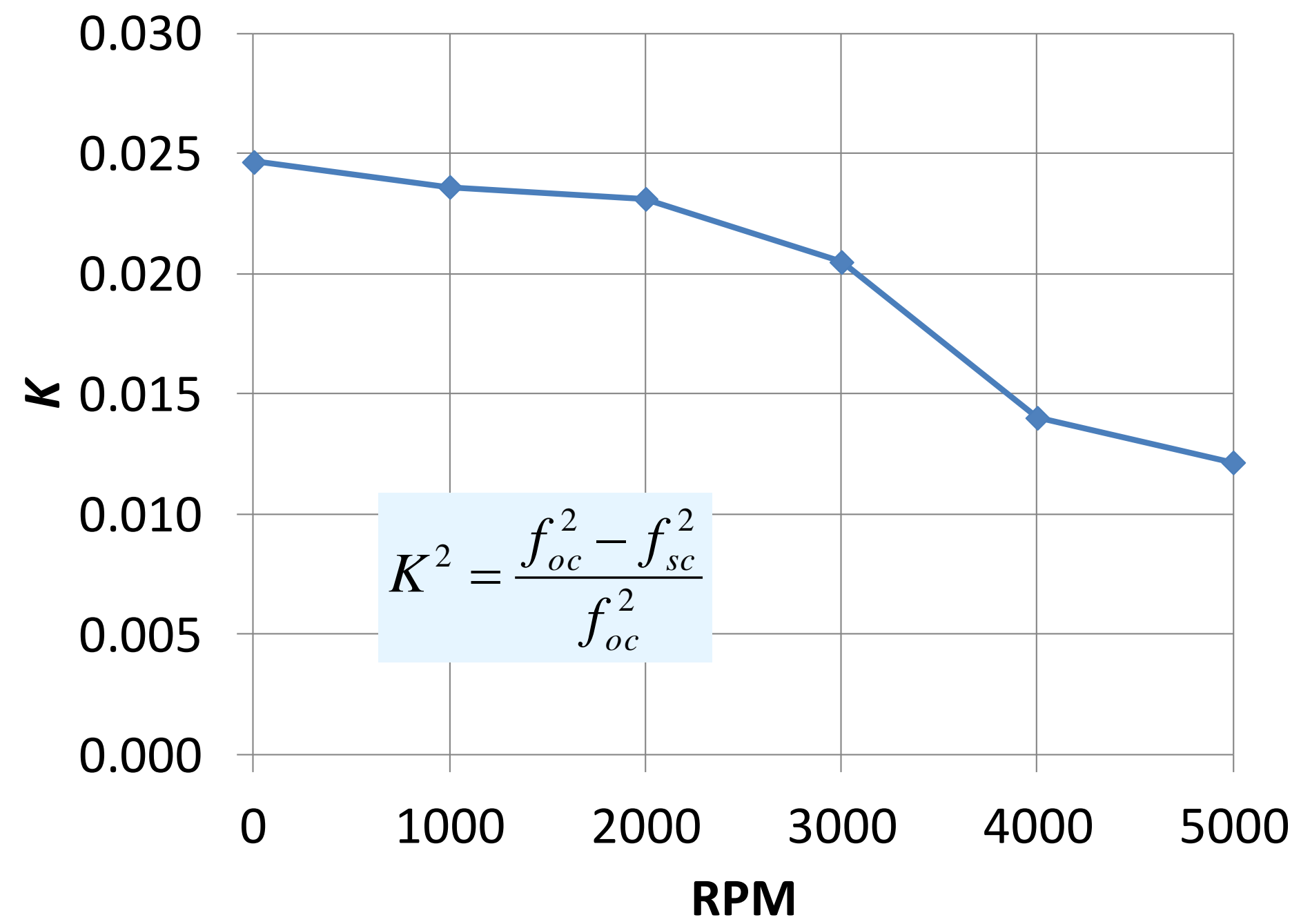




\section{Piezoelectric Patch as Sensor}

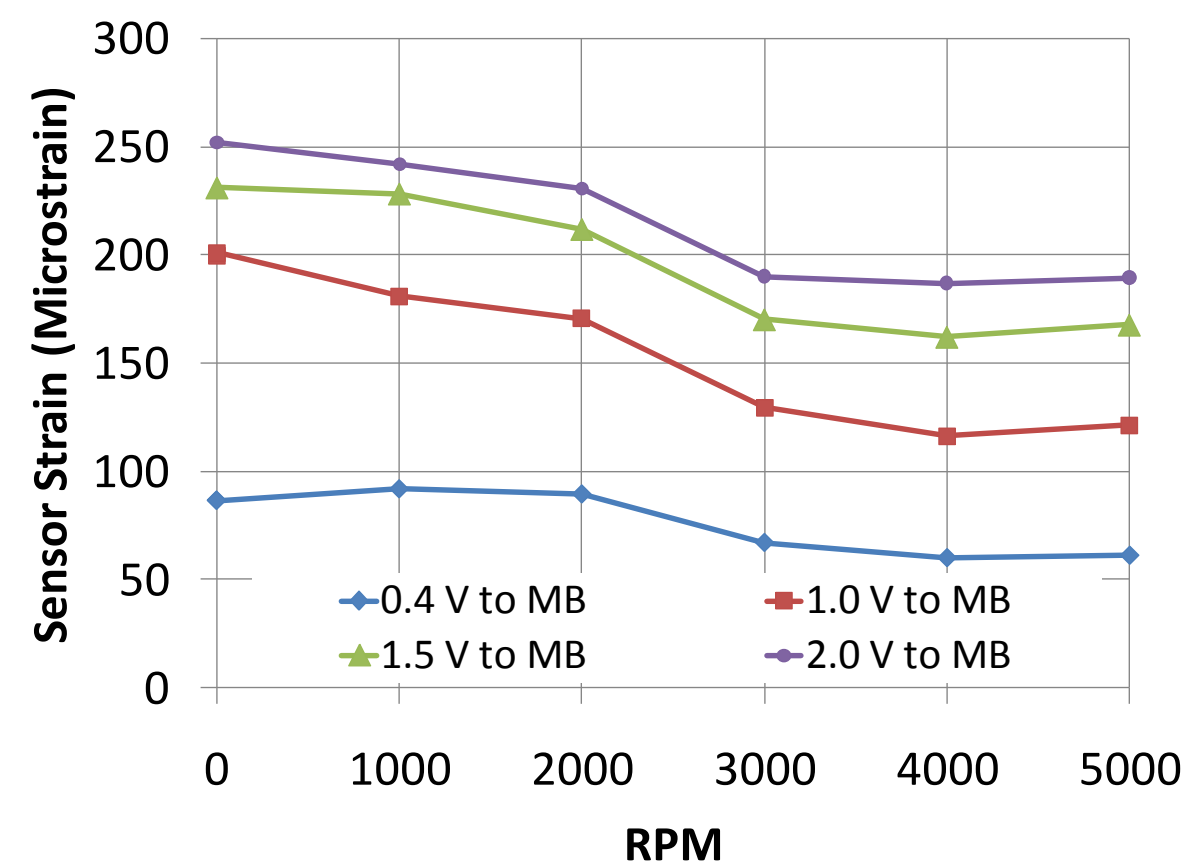

- Piezoelectric sensor voltage output proportional to strain

- Average strain over sensor area
- Excitation provided by magnetic bearings

- Strain measured by piezoelectric sensor

- Strain should be proportional to $K$

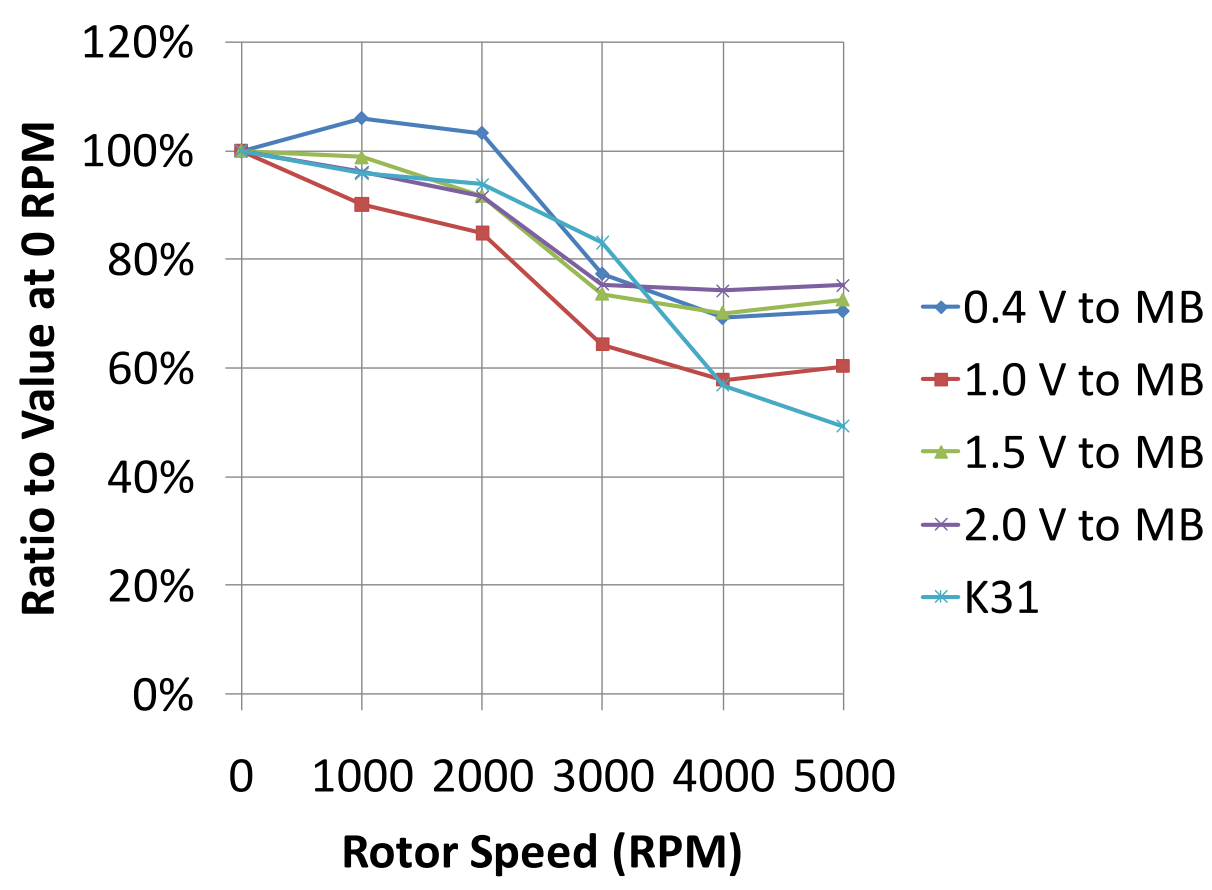




\section{Piezoelectric Patch as Actuator}

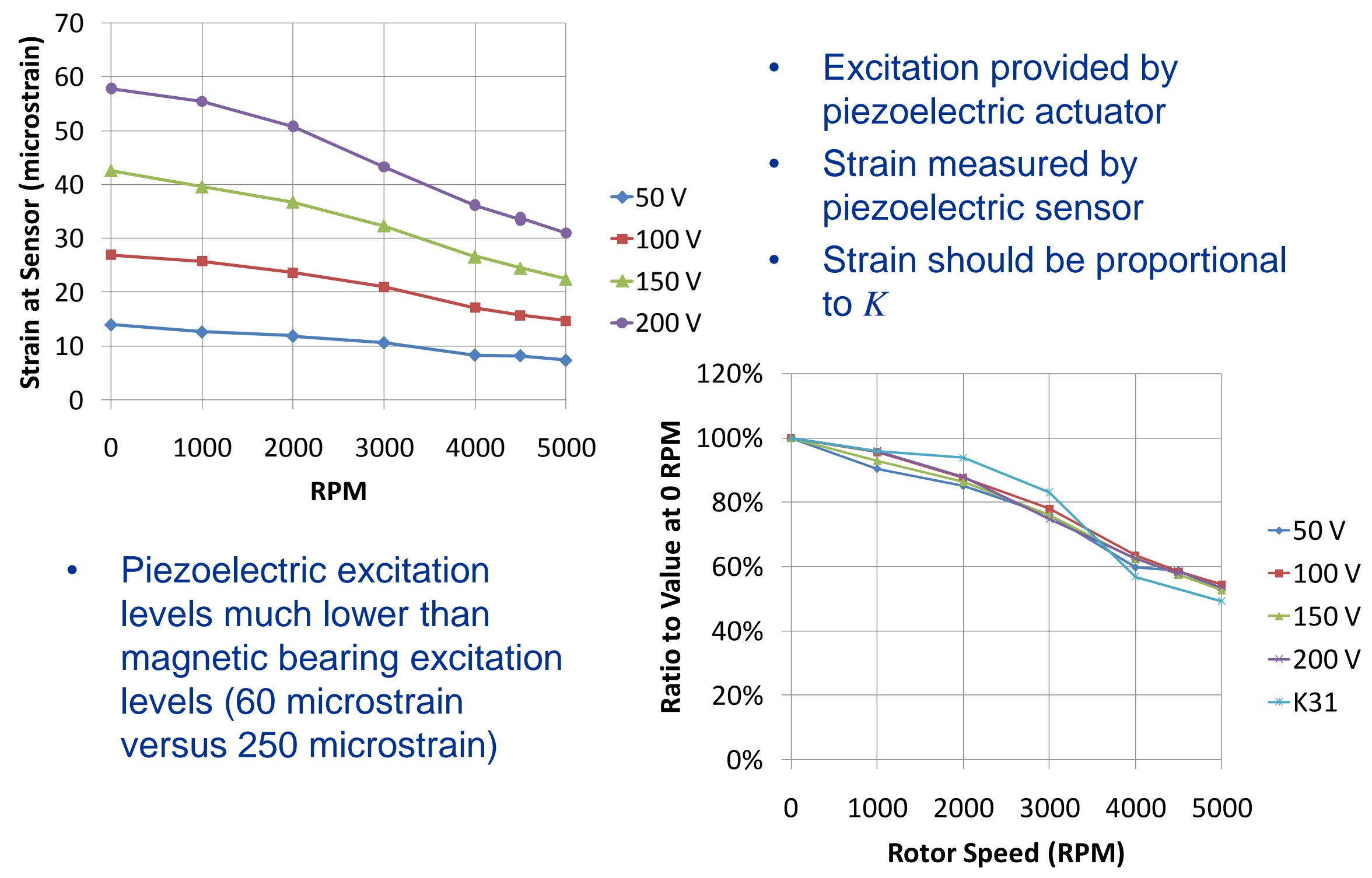




\section{Open Loop Vibration Control}

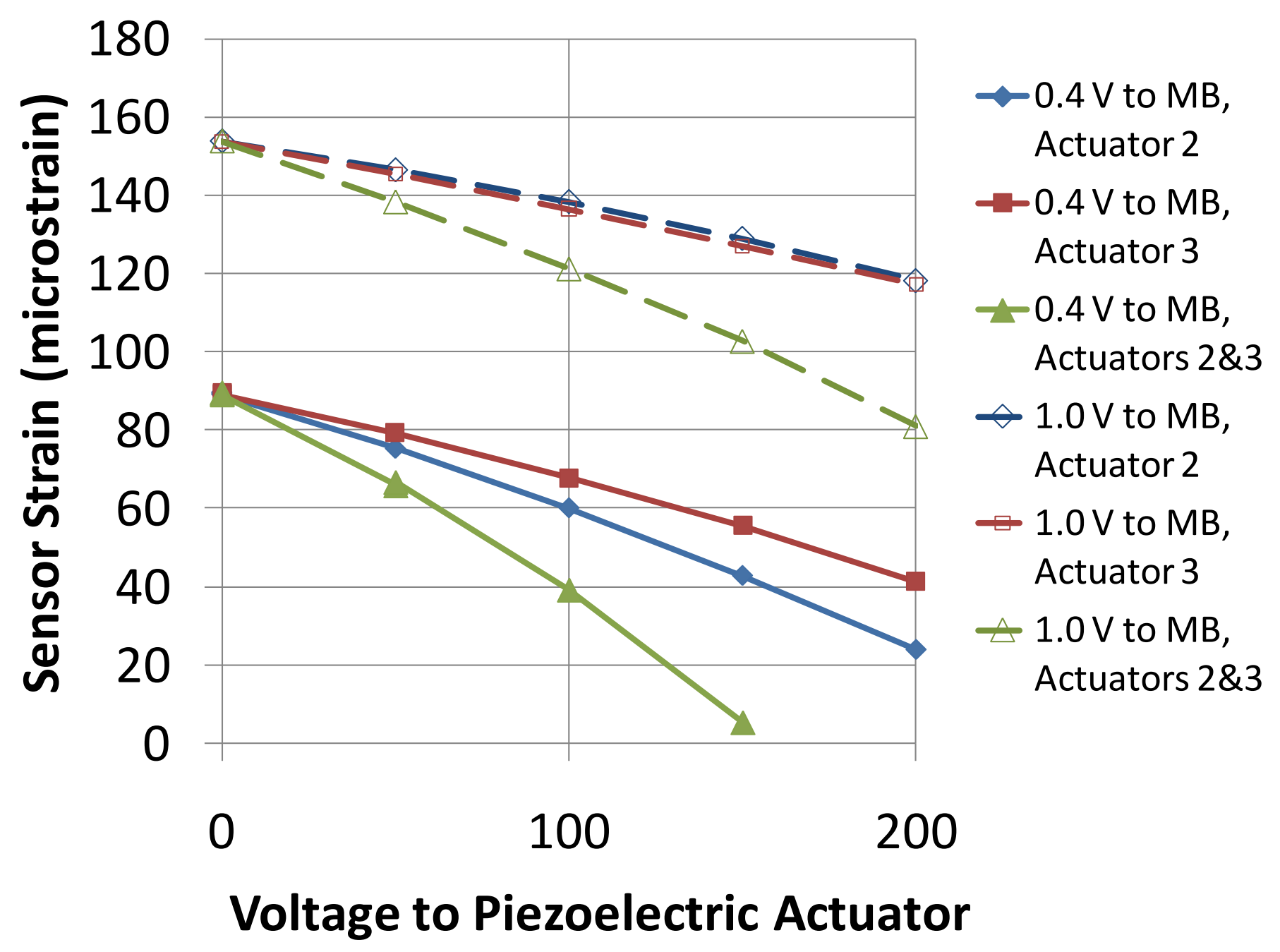




\section{Damping from Closed Loop Control}

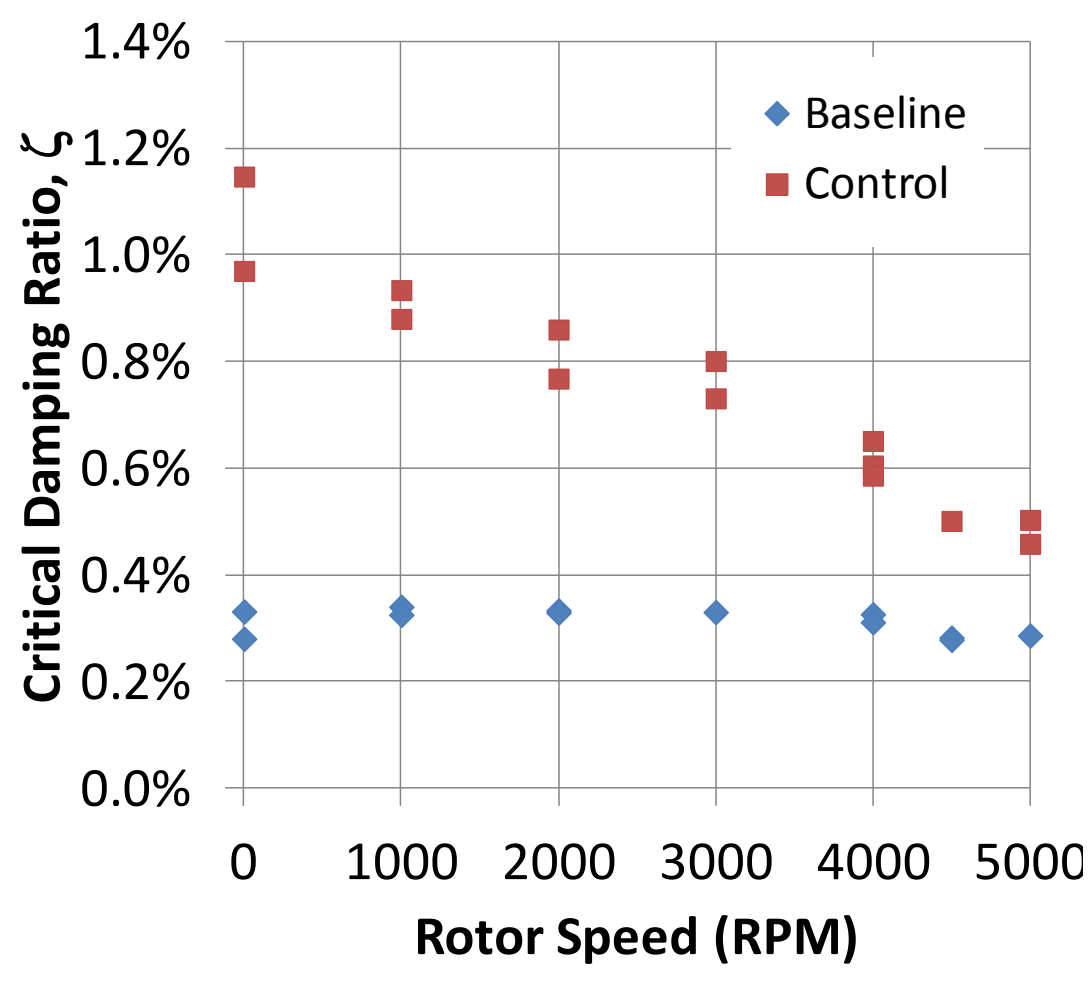

- Control system is simulated RLC circuit with amplification

- $\mathrm{R}=2500 \Omega$, bandwidth $\sim 4 \mathrm{~Hz}$

- L changes with blade frequency

- Low excitation level - voltage to actuators below max allowable

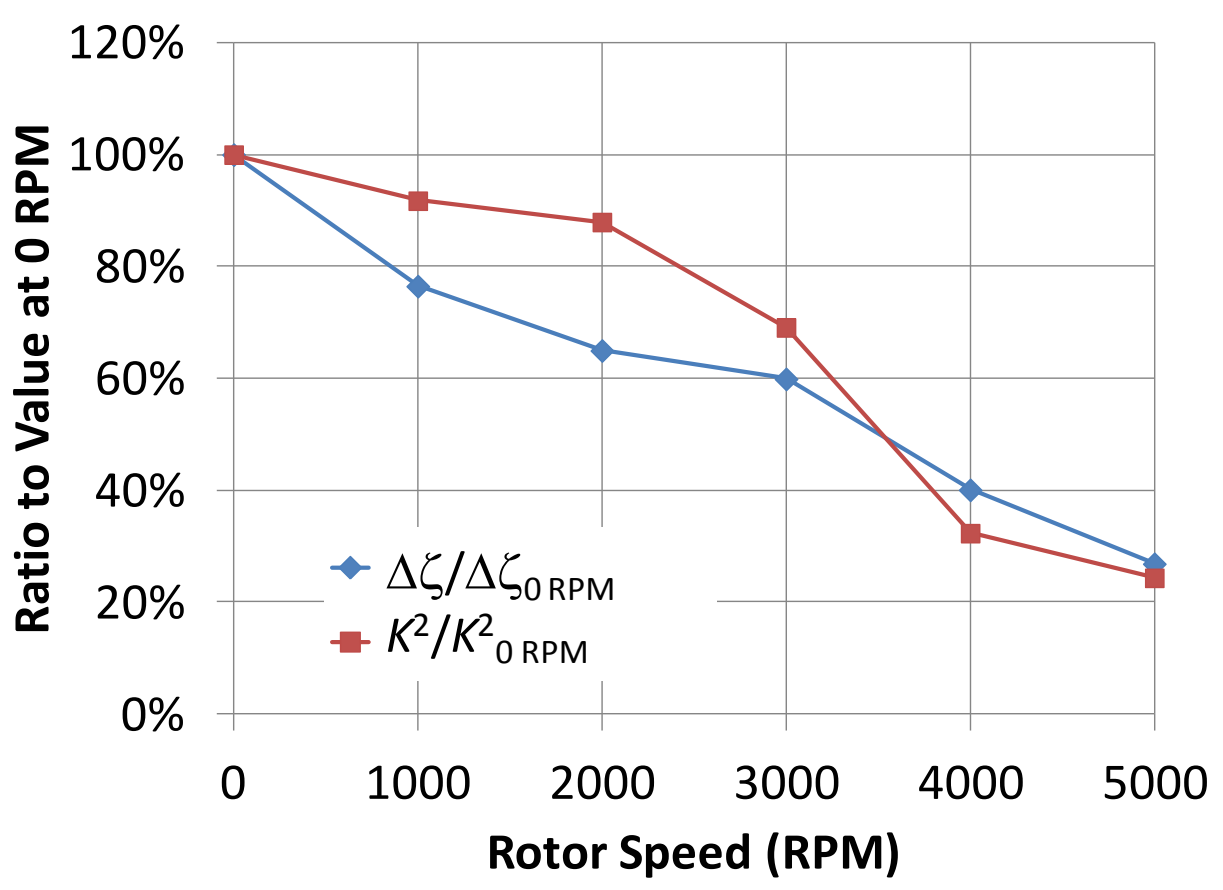




\section{Conclusions}

- Spin test conclusions

- Successful demonstration of open and closed loop control of blade vibration over a rotational speed range

- Up to $1 \%$ damping at 0 RPM, $0.5 \%$ damping at 5000 RPM

- Piezoelectric patches operated as designed under centrifugal and vibrational load

- Damping shown to be proportional to $K^{2}$

- Maximize $K$ to maximize damping

- Effect of target resonance mode

- $K$ is proportional to piezoelectric material elastic modulus and thickness (to first order)

- $K$ is proportional to material electromechanical coupling, $k$

- Single crystal material

- $d_{33}$ versus $d_{31}$ type actuators

- Optimize coverage area 


\section{Complementary Research at NASA GRC}

- Composites with embedded piezoelectric materials component strength and fatigue properties

- Material coupon testing (Duffy 2012)

- Subscale composite fan blades with embedded piezoelectric sensors and actuators

- Blades currently being fabricated - vibration testing

- Piezoelectric material property variation with temperature

- New material compositions

- Power transmission to piezoelectric actuators from the stationary frame

- Collaboration with Mesa Systems Co. to develop inductive power transmission device 


\section{Acknowledgments}

- NASA Fundamental Aeronautics Program, Subsonic Fixed Wing Project

- Space Act Agreement - NASA Glenn Research Center and GE Aviation

- Thanks to Jeff Kauffman (Penn State University) and Carlos Morrison (NASA Glenn) for their assistance during testing 